\title{
Dispersões Aquosas Poliuretânicas à Base de Polibutadieno Hidroxilado: Influência das Variáveis Reacionais
}

\author{
Lúcio C. de Magalhães \\ Petroflex Ltda., RJ
}

\author{
Fernanda M. B. Coutinho, Marcia C. Delpech \\ Instituto de Química, UERJ
}

\begin{abstract}
Resumo: Neste trabalho foram produzidas dispersões aquosas poliuretânicas com formulações variadas, em ausência de solvente orgânico. Nas sínteses, foram empregados o diisocianato de isoforona (IPDI) e como polióis, o poli(glicol propilênico) (PPG) e diferentes tipos de polibutadieno líquido hidroxilado (HTPB) comerciais. Para promover a dispersão das cadeias poliméricas em água, foi utilizado o ácido dimetilolpropiônico (DMPA), que após neutralização com trietilamina (TEA) forneceu os sítios iônicos responsáveis pela estabilidade das partículas dispersas no meio aquoso. Como extensor de cadeia foi utilizada a hidrazina (HYD). As dispersões aquosas poliuretânicas obtidas foram avaliadas quanto ao teor de sólidos totais, tamanho médio de partícula e viscosidade aparente. Não foram observadas variações significativas nesses valores, que ficaram em faixas pequenas, independentemente da formulação. Os filmes formados a partir dessas dispersões foram avaliados quanto às suas características visuais, adesão e propriedades mecânicas. Os filmes apresentaram boa aderência a superfícies de alumínio, transparência e flexibilidade e aqueles produzidos com baixos teores de DMPA e/ou baixa razão $\mathrm{NCO} / \mathrm{OH}$ apresentaram melhores propriedades mecânicas.
\end{abstract}

Palavras-chave: Poliuretanos, síntese, dispersões aquosas, polibutadieno líquido hidroxilado, diisocianato de isoforona.

\section{Development of Polyurethane Aqueous Dispersions Based on Hydroxy-terminated Polybutadiene: Reaction Variables}

Abstract: In this work, polyurethane aqueous dispersions with different formulations were produced in the absence of organic solvents. In the synthesis, isophorone diisocyanate (IPDI), poly(propylene glycol) (PPG) and different types of hydroxy-terminated polybutadiene (HTPB) were employed. In order to make possible the dispersion of the polyurethane chains in water, dimethylolpropionic acid (DMPA), was used. Afterwards the acid groups were neutralized with triethylamine (TEA) to provide the ionic groups responsible for the particles stability in water. The solids content, viscosity and particle size of the formulations produced in this work were evaluated. These parameters were found to vary within a narrow range, regardless of the formulation. The films were transparent, flexible and showed a good adherence on aluminum surfaces. Their mechanical properties were satisfactory, particularly those obtained with higher contents of DMPA and at low values of $\mathrm{NCO} / \mathrm{OH}$ ratio.

Keywords: Polyurethanes, synthesis, aqueous dispersions, hydroxyl-terminated polybutadiene, isophorone diisocianate.

\section{Introdução}

Nos últimos anos, as questões ambientais têm merecido um papel de destaque na mídia nacional e internacional. Os problemas do meio ambiente têm estado presentes em praticamente todas as reuniões de Chefes de Estado, seja em relação à redução na emissão de compostos orgânicos voláteis (VOC's), no controle da degradação de reservas ambientais, ou no desenvolvimento auto-sustentável (DS ${ }^{[1,2]}$. Dessa forma, a produção de sistemas químicos menos agressivos tem sido o foco de muitos trabalhos de pesquisa ${ }^{[3-14]}$.

A síntese de polímeros dispersos em água constitui um verdadeiro avanço tecnológico, principalmente na área de materiais para revestimento, onde tradicionalmente são empregadas soluções em solventes orgânicos ${ }^{[3-16]}$. Neste último caso, após a aplicação da solução ocorre a evaporação do solvente orgânico para o meio ambiente, o que é, atualmente, proibido na Europa, por exemplo. Dentre as dispersões aquosas poliméricas, as que mais têm se destacado são aquelas à base de poliuretanos. Isso se deve à grande possibilidade de combinações entre os monômeros, resultando em cadeias com as mais variadas características e propriedades, dando origem a termoplásticos, termorrígidos, elastômeros ou fibras. Os poliuretanos são aplicados, por exemplo, como adesivos ou revestimentos para diversos tipos de substratos em vários segmentos industriais, tais como: automotivo, aeroespacial, moveleiro, têxtil e calçadista ${ }^{[17,18]}$.

Os poliuretanos, de um modo geral, assim como a maioria dos polímeros sintéticos, não são compatíveis com a

Autor para correspondência: Fernanda M. B. Coutinho, Departamento de Processos Químicos, Instituto de Química, UERJ, Av. Maracanã 524, CEP: 20559-900, Rio de Janeiro, RJ, Brasil. E-mail: fern@ajato.com.br 
água. Assim, é necessário promover modificações na estrutura da cadeia poliuretânica, com a inserção de grupos ou segmentos que tenham afinidade com o meio aquoso. Isso pode ser conseguido com a introdução de grupos iônicos, que podem ser provenientes dos monômeros iniciais ou de um extensor de cadeia. A presença desses grupos, associada ao tamanho médio das partículas adequado, leva à formação de dispersões aquosas estáveis ${ }^{[3-14]}$.

O objetivo deste trabalho foi a produção de dispersões aquosas poliuretânicas, de natureza aniônica, à base de polibutadieno hidroxilado (HTPB). A característica hidrofóbica da cadeia do HTPB dificulta a dispersão do poliuretano em água e, por essa razão, são encontrados poucos estudos na literatura sobre esses sistemas. Neste trabalho, foram sintetizados poliuretanos com três diferentes tipos de HTPB em proporções variadas, sendo usado um isocianato de natureza alifática, o diisocianato de isoforona (IPDI).

\section{Experimental}

\section{Materiais}

Os reagentes utilizados nas sínteses das dispersões aquosas poliuretânicas foram usados como recebidos. Dois tipos de polióis foram utilizados: 1. Poli(glicol propilênico) (PPG) - Voranol 2120, de massa molar numérica média igual a $2000 \mathrm{~g} / \mathrm{mol}$ e número de hidroxilas $=50,83 \mathrm{mg} \mathrm{KOH} / \mathrm{g}$ de poliol $^{[19]}$. 2. Polibutadieno líquido hidroxilado(HTPB): Liquiflex $\mathrm{H}$, de massa molar numérica média igual a $2400 \mathrm{~g} / \mathrm{mol}$ e número de hidroxilas $=46,17 \mathrm{mg} \mathrm{KOH} / \mathrm{g}$ de poliol, Liquiflex $\mathrm{P}$, de massa molar numérica média igual a $2700 \mathrm{~g} / \mathrm{mol}$ e número de hidroxilas $=43,48 \mathrm{mg} \mathrm{KOH} / \mathrm{g}$ de poliol ${ }^{[20]}$, e R-45HTLO, de massa molar numérica média igual a $2200 \mathrm{~g} / \mathrm{mol}$ e número de hidroxilas $=43,48 \mathrm{mg} \mathrm{KOH} / \mathrm{g}$ de poliol ${ }^{[21]}$. Como diisocianato escolheu-se o de isoforona (IPDI), de natureza alifática, mais resistente à oxidação por radiação ultravioleta. O ácido dimetilolpropiônico (DMPA) foi o gerador de sítios aniônicos e a trietilamina (TEA) foi o agente neutralizante para as carboxilas do DMPA. A hidrazina (HYD), que foi o extensor de cadeia, deu origem a poli(uretano-uréia)s.

\section{Síntese}

As dispersões aquosas poliuretânicas foram produzidas por um processo em duas etapas pelo método do prepolímero em massa, ou seja, em ausência de solvente. A primeira etapa da síntese foi conduzida em duas sub-etapas: síntese do prepolímero e neutralização das carboxilas derivadas do DMPA. Os monômeros utilizados na primeira etapa foram: poli(glicol propilênico) (PPG), polibutadieno líquido hidroxilado (HTPB), ácido dimetilolpropiônico (DMPA) e diisocianato de isoforona (IPDI). Na segunda etapa, foi feita a dispersão da massa polimérica em água, seguida da extensão de cadeia por meio de reação com hidrazina (HYD).

As sínteses foram conduzidas em atmosfera não-iner$\mathrm{te}^{[5,7,9,12]} \mathrm{em}$ um reator do tipo kettle, onde foram adicionados os polióis (PPG + HTPB), o monômero gerador de sítios iônicos (DMPA) e o diisocianato de isoforona (IPDI). As reações de obtenção dos prepolímeros foram conduzidas por cerca de 1 hora, a uma temperatura em torno de $100{ }^{\circ} \mathrm{C}$, com velocidade de agitação próxima a 1000 rpm. Posteriormente, as carboxilas, oriundas do DMPA, foram neutralizadas com trietilamina (TEA), a uma temperatura inferior a $45^{\circ} \mathrm{C}$, por $30 \mathrm{~min}$, com velocidade de agitação próxima a $1000 \mathrm{rpm}$.

A dispersão em água da massa polimérica foi realizada a uma velocidade de agitação em torno de 2000 rpm, sendo mantida nesse patamar por aproximadamente 40 minutos. Essa velocidade variou em função da viscosidade do prepolímero. Para meios reacionais mais viscosos a velocidade era maior. Em seguida, foi realizada a extensão de cadeia por meio de reação do prepolímero disperso, com hidrazina, por 30 minutos, a temperatura de aproximadamente $35^{\circ} \mathrm{C}$. Vale ressaltar que todas as dispersões foram produzidas sem o auxílio de solventes ou catalisadores.

Os cálculos das formulações foram feitos de modo a se obter dispersões com teor de sólidos de 35\%. Os seguintes parâmetros reacionais foram variados: o tipo do polibutadieno líquido hidroxilado (HTPB) (Liquiflex H, Liquiflex P e R-45HTLO); o percentual de HTPB na formulação, em termos de equivalentes gramas, em 0,10 e $30 \%$, em relação ao percentual total de poliol (onde: poliol $=$ HTPB + PPG); o percentual de ácido dimetilolpropiônico (DMPA) em termos de equivalentes-grama, em 50 e 70\%, em relação ao total de compostos hidroxilados (DMPA + Poliol); e a razão entre o número de equivalentes-grama de grupos isocianato e hidroxilas $(\mathrm{NCO} / \mathrm{OH})$, em 2, 3 e 4 . As Tabelas 1 e 2 mostram as variações feitas nas formulações, designadas como Séries 1 e 2 , respectivamente.

Tabela 1. Formulações da Série 1.

\begin{tabular}{cccc}
\hline $\begin{array}{c}\text { Relação } \\
\text { DMPA/Poliol } \\
(\% / \%)\end{array}$ & $\begin{array}{c}\text { Relação } \\
\text { PPG/HTPB } \\
(\boldsymbol{\%} / \%)\end{array}$ & $\begin{array}{c}\text { Tipo de } \\
\text { HTPB }\end{array}$ & $\begin{array}{c}\text { Razão } \\
\text { NCO/OH }\end{array}$ \\
\hline $70 / 30$ & $100 / 0$ & - & 2 \\
$70 / 30$ & $70 / 30$ & Liquiflex P & 2 \\
$70 / 30$ & $90 / 10$ & Liquiflex P & 2 \\
$70 / 30$ & $70 / 30$ & Liquiflex H & 2 \\
$70 / 30$ & $90 / 10$ & Liquiflex H & 2 \\
\hline
\end{tabular}

Poliol: PPG + HTPB.

Tabela 2. Formulações da Série 2.

\begin{tabular}{cccccc}
\hline $\begin{array}{c}\text { Relação } \\
\text { DMPA/Poliol } \\
(\% / \%)\end{array}$ & $\begin{array}{c}\text { Relação } \\
\text { PPG/HTPB } \\
(\% / \%)\end{array}$ & $\begin{array}{c}\text { Tipo de } \\
\text { HTPB }\end{array}$ & \multicolumn{2}{c}{$\begin{array}{c}\text { Razão } \\
\text { NCO/OH }\end{array}$} \\
\hline $70 / 30$ & $100 / 0$ & - & 2 & 3 & 4 \\
$70 / 30$ & $70 / 30$ & Liquiflex H & 2 & 3 & 4 \\
$70 / 30$ & $70 / 30$ & R-45HTLO & 2 & 3 & 4 \\
$50 / 50$ & $100 / 0$ & - & 2 & 3 & 4 \\
$50 / 50$ & $70 / 30$ & Liquiflex H & 2 & 3 & 4 \\
$50 / 50$ & $70 / 30$ & R-45HTLO & 2 & 3 & 4 \\
\hline
\end{tabular}

Poliol: PPG + HTPB. 


\section{Caracterização}

As dispersões poliuretânicas foram caracterizadas quanto ao teor de sólidos, o tamanho médio de partícula e a viscosidade aparente.

O teor de sólidos foi determinado algumas horas após o término de cada síntese, adicionando-se $1 \mathrm{~g}$ da dispersão em uma cápsula de alumínio, previamente tarada, que foi então colocada em uma estufa a vácuo $(200 \mathrm{~mm} / \mathrm{Hg})$, a $60{ }^{\circ} \mathrm{C}$, por 4 horas. Após a secagem, a massa residual foi relacionada à massa da dispersão inicial, sendo esta razão multiplicada por 100, para obtenção dos valores em percentual.

O tamanho médio de partícula foi determinado por espalhamento dinâmico de luz (DLS) em um equipamento Zetasizer Nano ZS, capaz de realizar a detecção de tamanhos de partículas em níveis de alta sensibilidade, na faixa de $0,6 \mathrm{~nm}$ a $6 \mu \mathrm{m}$. Os valores de tamanho médio de partícula (TMP) foram determinados, levando as dispersões aquosas produzidas à diluição infinita com água destilada.

A viscosidade aparente das dispersões foi avaliada em um viscosímetro Brookfield LVT, utilizando um spindle Yula 15 com um adaptador UL universal, com velocidade de $12 \mathrm{rpm}$ (taxa de cisalhamento de $14,68 \mathrm{~s}^{-1}$ ) e temperatura de $25,0 \pm 0,1{ }^{\circ} \mathrm{C}$.

A partir das dispersões aquosas, foram preparados filmes por vazamento dessas dispersões em moldes de vidro revestidos com poli(tetrafluoroetileno) (PTFE), providos de lâminas de vidro nas laterais, formando barreiras, que impediam que a dispersão vazada escoasse para fora do molde. Após secagem na temperatura ambiente, por aproximadamente 7 dias, os filmes produzidos, com dimensões de $15 \times 15,5 \mathrm{~cm}$ e espessuras na faixa de 0,20 a $0,35 \mathrm{~mm}$, foram avaliados quanto à resistência mecânica.

As propriedades mecânicas de tensão na ruptura e alongamento na ruptura foram determinadas em Máquina Universal de Ensaios Instron com garras pneumáticas, modelo 5565 e célula de carga de $1 \mathrm{kN}$, como valores medianos obtidos a partir da determinação de cinco corpos de prova cortados sob a forma de "gravatinhas", conforme determinado na metodologia ISO 37, com as seguintes dimensões: comprimento total $=75 \mathrm{~mm}$, largura nas extremidades $=12,5 \mathrm{~mm}$, comprimento da porção estreita $=4 \mathrm{~mm}$, raio pequeno $=8 \mathrm{~mm}$, raio grande $=12,5 \mathrm{~mm}$, espessura $=2 \mathrm{~mm}$. A velocidade de separação das garras foi de $500 \mathrm{~mm} / \mathrm{min}^{[22]}$.

As dispersões aquosas foram aplicadas nos seguintes substratos: poli(tetrafluoroetileno) (PTFE), no qual os filmes citados anteriormente foram obtidos; em filmes de polietileno (PE), e em folhas de alumínio (Al). Os revestimentos formados, após secagem, foram avaliados de forma qualitativa, quanto a características como cor, translucidez, dureza e aderência aos substratos testados. A observação das propriedades na superfície de PTFE foi feita diretamente nos filmes produzidos para os testes de propriedades mecânicas. Sua maior espessura $(0,20$ a $0,35 \mathrm{~mm})$ permitiu observar um ligeiro amarelecimento do produto, que não era possível detectar nos filmes finos vazados sobre os outros substratos. Para avaliar a aderência sobre a superfície de alumínio e polietileno, as dispersões foram vazadas e espalhadas sobre essas superfícies, de modo a formar uma fina película após a secagem.

\section{Resultados e Discussão}

Foram preparadas 23 diferentes formulações de dispersões aquosas poliuretânicas, todas produzidas em duplicata. Os resultados apresentados são valores médios de determinações obtidas em duas reações, realizadas com formulações e parâmetros reacionais idênticos.

\section{Caracterização das dispersões aquosas}

\section{Sólidos totais}

Foi observado que, no preparo do prepolímero, a viscosidade do meio reacional variou de acordo com a massa de HTPB e/ou diisocianato adicionados ao sistema, o que influenciou na viscosidade do meio. Quanto maior o teor de HTPB e menor a razão $\mathrm{NCO} / \mathrm{OH}$, maior a viscosidade do prepolímero e mais difícil a dispersão da massa reacional em água. O caráter hidrofóbico do HTPB, certamente, também tem influência nessa etapa, mas não a impossibilita.

No entanto, foi observado que, independente da formulação, a perda de sólidos não-dispersos foi mínima já que o teor de sólidos obtido variou na faixa de 32 a $34 \%$ para todas as formulações e respectivas duplicatas.

\section{Tamanho médio de partícula}

As Tabelas 3 e 4 apresentam os resultados do tamanho médio de partícula das formulações das Séries 1 e 2, respectivamente. Pode-se observar que os tamanhos de partícula apresentaram pequenas variações, a maioria dentro da mesma ordem de grandeza.

As medidas de tamanho médio de partícula (TMP) foram determinadas levando as dispersões aquosas produzidas à diluição infinita com água destilada. Avaliando os resultados obtidos na Série 1, pode-se observar que, para as formulações com o mesmo tipo de HTPB, o tamanho médio de partículas das formulações com maior teor (30\%) foram mais elevados do que os das formulações com menor teor (10\%). Esses resultados mostram que a maior viscosidade do prepolímero, provocada por maiores quantidades de HTPB, dificultando a dispersão, levou à formação de partículas maiores. A síntese

Tabela 3. Valores de tamanho médio de partícula (TMP) das dispersões aquosas poliuretânicas da Série 1.

\begin{tabular}{|c|c|c|c|}
\hline \multirow{2}{*}{$\begin{array}{c}\text { Relação } \\
\text { DMPA/Poliol } \\
(\% / \%)\end{array}$} & \multirow{2}{*}{$\begin{array}{c}\text { Relação } \\
\text { HTPB/PPG } \\
(\% / \%)\end{array}$} & \multirow{2}{*}{$\begin{array}{l}\text { Tipo de } \\
\text { HTPB }\end{array}$} & $\mathrm{NCO} / \mathrm{OH}=2$ \\
\hline & & & TMP ( nm ) \\
\hline $70 / 30$ & $0 / 100$ & - & 118 \\
\hline $70 / 30$ & $10 / 90$ & Liquiflex $\mathrm{H}$ & 99 \\
\hline $70 / 30$ & $30 / 70$ & Liquiflex $\mathrm{H}$ & 127 \\
\hline $70 / 30$ & $10 / 90$ & Liquiflex P & 101 \\
\hline $70 / 30$ & $30 / 70$ & Liquiflex P & 170 \\
\hline
\end{tabular}


Tabela 4. Valores de tamanho médio de partícula (TMP) das dispersões aquosas poliuretânicas da Série 2.

\begin{tabular}{|c|c|c|c|c|c|}
\hline \multirow{2}{*}{$\begin{array}{c}\text { Relação } \\
\text { DMPA/Poliol } \\
(\% / \%)\end{array}$} & \multirow{2}{*}{$\begin{array}{c}\text { Relação } \\
\text { HTPB/PPG } \\
(\% / \%)\end{array}$} & \multirow[t]{2}{*}{ Tipo de HTPB } & \multicolumn{3}{|c|}{ TMP (nm) } \\
\hline & & & $\mathrm{NCO} / \mathrm{OH}=2$ & $\mathrm{NCO} / \mathrm{OH}=3$ & $\mathrm{NCO} / \mathrm{OH}=4$ \\
\hline $70 / 30$ & $0 / 100$ & - & 118 & 133 & 85 \\
\hline $70 / 30$ & $30 / 70$ & Liquiflex H & 127 & 131 & 139 \\
\hline $70 / 30$ & $30 / 70$ & R-45HTLO & 147 & 118 & 118 \\
\hline $50 / 50$ & $0 / 100$ & - & 71 & 89 & ND \\
\hline $50 / 50$ & $30 / 70$ & Liquiflex H & 146 & 123 & 123 \\
\hline $50 / 50$ & $30 / 70$ & R-45HTLO & 146 & 144 & 131 \\
\hline
\end{tabular}

ND- Não determinado. Sedimentação 24 h após a síntese.

realizada com 30\% do HTPB de maior massa molar (Líquiflex $P, \bar{M} n=2700$ ) apresentou um valor de TMP significativamente maior do que os encontrados para as outras formulações. Isso confirma a dificuldade provocada pela presença da HTPB na dispersão do prepolímero.

Avaliando-se os resultados da Série 2, pode-se observar que há uma tendência à redução nos valores de tamanho de partícula à medida que a razão $\mathrm{NCO} / \mathrm{OH}$ vai aumentando. Razões $\mathrm{NCO} / \mathrm{OH}$ maiores implicam na presença de um maior número de ligações uretânicas e urêicas, conferindo maior caráter hidrofílico às cadeias, o que pode ter facilitado a formação das partículas no momento da dispersão.

A ausência de HTPB na formulação, como era de esperar, provocou uma tendência à diminuição da viscosidade do prepolímero e redução no tamanho das partículas ${ }^{[7]}$. A diferença entre as massas molares dos dois tipos de HTPB (Liquiflex $\mathrm{H}: \overline{\mathrm{Mn}}=2400 \mathrm{~g} / \mathrm{mol}$ e R-45HTLO: $\overline{\mathrm{M}} \mathrm{n}=2200 \mathrm{~g} / \mathrm{mol}$ ) não promoveu um comportamento diferenciado.

De acordo com a literatura, quanto maior a hidrofilicidade das partículas, menor será o seu tamanho ${ }^{[23-24]}$. No entanto, os resultados da Tabela 4 mostram que maiores teores de DMPA na formulação (50/50) não influenciaram de forma marcante os valores de tamanho de partícula e não promoveram um comportamento padronizado em função da razão $\mathrm{NCO} / \mathrm{OH}$.

Para as formulações com relação DMPA/Poliol $=50 / 50$, não foi possível obter nem mesmo uma dispersão estável, em ausência de HTPB. Isso pode ser atribuído ao alto teor de diisocianato associado ao menor teor de DMPA empregado na composição, que aumentou o teor de segmentos rígidos ${ }^{[17-18]}$ desestabilizando a dispersão.
Tabela 5. Viscosidade aparente das dispersões da série 1

\begin{tabular}{|c|c|c|c|}
\hline Relação & Relação & Tipo de & Razão 2 \\
\hline $\begin{array}{c}\text { DMPA/Poliol } \\
(\% / \%)\end{array}$ & $\begin{array}{c}\text { HTPB/PPG } \\
(\% / \%)\end{array}$ & НТРВ & $\begin{array}{c}\begin{array}{l}\text { Viscosidade } \\
(\mathrm{mPa} . \mathrm{s})\end{array} \\
\end{array}$ \\
\hline $70 / 30$ & $0 / 100$ & - & 13 \\
\hline $70 / 30$ & $10 / 90$ & Liquiflex $\mathrm{H}$ & 15 \\
\hline $70 / 30$ & $30 / 70$ & Liquiflex $\mathrm{H}$ & 16 \\
\hline $70 / 30$ & $10 / 90$ & Liquiflex $\mathrm{P}$ & 16 \\
\hline $70 / 30$ & $30 / 70$ & Liquiflex $\mathrm{P}$ & 17 \\
\hline
\end{tabular}

\section{Viscosidade aparente}

As Tabelas 5 e 6 apresentam os resultados de viscosidade aparente das dispersões produzidas nas Séries 1 e 2, respectivamente.

Os resultados das Tabelas 5 e 6 mostram que não houve variação significativa nos valores de viscosidade, independente da formulação. Pode-se observar uma tendência à redução nas viscosidades das dispersões obtidas em ausência de HTPB e para razões NCO/OH maiores, ou seja, dispersões obtidas a partir de prepolímeros menos viscosos e com caráter mais hidrofílico.

A presença de maiores teores de DMPA também levou a uma redução na viscosidade, exceto para a razão $\mathrm{NCO} / \mathrm{OH}$ mais baixa, na qual o caráter hidrofílico diminui em função do menor teor de ligações uretano e uréia.

Apesar de todas as variações feitas nas formulações e dos fatores sinérgicos entre elas, os resultados mostram que, independente da composição das dispersões aquosas, os valores de viscosidade aparente foram baixos, o que é extremamente vantajoso em termos de facilidade na aplicação das dispersões como revestimentos.

Tabela 6. Viscosidade aparente das dispersões da Série 2.

\begin{tabular}{|c|c|c|c|c|c|}
\hline \multirow{2}{*}{$\begin{array}{c}\text { Relação } \\
\text { DMPA/Poliol } \\
(\% / \%)\end{array}$} & \multirow{2}{*}{$\begin{array}{c}\text { Relação } \\
\text { HTPB/PPG } \\
(\% / \%)\end{array}$} & \multirow[t]{2}{*}{ Tipo de НTPB } & \multicolumn{3}{|c|}{ Viscosidade (mPa.s) } \\
\hline & & & $\mathrm{NCO} / \mathrm{OH}=2$ & $\mathrm{NCO} / \mathrm{OH}=3$ & $\mathrm{NCO} / \mathrm{OH}=4$ \\
\hline $30 / 70$ & $0 / 100$ & - & 13 & 17 & 17 \\
\hline $30 / 70$ & $30 / 70$ & Liquiflex $\mathrm{H}$ & 16 & 14 & 19 \\
\hline $30 / 70$ & $30 / 70$ & R-45HTLO & 14 & 15 & 16 \\
\hline $50 / 50$ & $0 / 100$ & - & 17 & 8 & ND \\
\hline $50 / 50$ & $30 / 70$ & Liquiflex $\mathrm{H}$ & 19 & 8 & 7 \\
\hline $50 / 50$ & $30 / 70$ & R-45HTLO & 17 & 10 & 9 \\
\hline
\end{tabular}

ND- Não determinado. Sedimentação 24 h após a síntese. 
Os valores de viscosidade observados para as dispersões não mostraram uma associação padronizada com os valores de tamanho médio de partícula. O que se pode concluir, relacionando-se os resultados obtidos por ambas as técnicas, é que os valores de viscosidade aparente e de tamanho de partícula variaram em faixas pequenas, independente da formulação.

\section{Caracterização dos Filmes e Revestimentos}

\section{Características visuais e de aderência dos revestimentos}

As Tabelas 7 (Série 1) e 8 (Série 2) apresentam informações relacionadas às características visuais e de adesão dos revestimentos obtidos após aplicação das dispersões aquosas em substratos de poli(tetrafluoroetileno) (PTFE), polietileno (PE) e alumínio (Al).
Os resultados mostram que os revestimentos obtidos não apresentaram aderência ao PTFE e ao PE, mas aderiram fortemente às folhas de alumínio. A não-aderência dos filmes de poliuretano às superfícies de PTFE e de PE pode ser atribuída à grande diferença de polaridade existente entre os filmes (predominantemente polares) e os substratos (apolares). A aderência dos filmes de poliuretano ao alumínio pode ser atribuída ao fato de que as superfícies metálicas são boas aceptoras de elétrons (ácido de Lewis), e que a estrutura molecular dos filmes de poliuretano possuem átomos de nitrogênio e de oxigênio com um par de elétrons livres (base de Lewis), que interagem fortemente com os orbitais vazios da superfície metálica. A Figura 1 mostra uma representação esquemática da interação entre o filme de poliuretano e o substrato de alumínio. Isso explica também a falta de aderência

Tabela 7. Características visuais e de adesão observadas nos revestimentos obtidos a partir da aplicação das dispersões da Série 1 em superfícies de poli(tetrafluoroetileno) (PTFE), polietileno (PE) e alumínio (Al).

\begin{tabular}{|c|c|c|c|c|c|c|c|c|}
\hline \multirow{2}{*}{$\begin{array}{c}\text { Relação } \\
\text { DMPA/Polióis } \\
(\% / \%)\end{array}$} & \multirow{2}{*}{$\begin{array}{c}\text { Relação } \\
\text { HTPB/PPG } \\
(\% / \%)\end{array}$} & \multirow{2}{*}{$\begin{array}{l}\text { Tipo de } \\
\text { HTPB }\end{array}$} & \multicolumn{3}{|c|}{ Características visuais } & \multicolumn{3}{|c|}{ Características de aderência } \\
\hline & & & Coloração & Dureza deade & Translucidez & PTFE & $\mathbf{P E}$ & Al \\
\hline $70 / 30$ & $0 / 100$ & - & incolor* & flexível & transparente & não & não & $\operatorname{sim}$ \\
\hline $70 / 30$ & $10 / 90$ & Liquiflex $\mathrm{H}$ & incolor & flexível & transparente & não & não & $\operatorname{sim}$ \\
\hline $70 / 30$ & $30 / 70$ & Liquiflex $\mathrm{H}$ & incolor & flexível & transparente & não & não & $\operatorname{sim}$ \\
\hline $70 / 30$ & $10 / 90$ & Liquiflex P & incolor & flexível & transparente & não & não & $\operatorname{sim}$ \\
\hline $70 / 30$ & $30 / 70$ & Liquiflex P & incolor & flexível & transparente & não & não & $\operatorname{sim}$ \\
\hline
\end{tabular}

* ligeiramente amarelado.

Tabela 8. Características visuais e de adesão observadas nos revestimentos obtidos a partir da aplicação das dispersões da Série 2 em superfícies de poli(tetrafluoroetileno) (PTFE), polietileno (PE) e alumínio (Al).

\begin{tabular}{|c|c|c|c|c|c|c|c|c|}
\hline \multirow{2}{*}{$\begin{array}{c}\text { Relação } \\
\text { DMPA/Polióis } \\
(\% / \%)\end{array}$} & \multirow{2}{*}{$\begin{array}{c}\text { Relação } \\
\text { HTPB/PPG } \\
(\% / \%)\end{array}$} & \multirow[t]{2}{*}{ Tipo de HTPB } & \multicolumn{3}{|c|}{ Características visuais } & \multicolumn{3}{|c|}{ Características de aderência } \\
\hline & & & Cor & Dureza & Translucidez & PTFE & PE & Al \\
\hline \multicolumn{9}{|c|}{ Razão NCO/OH = 2} \\
\hline $30 / 70$ & $0 / 100$ & - & incolor* & flexível & transparente & não & não & $\operatorname{sim}$ \\
\hline $30 / 70$ & $30 / 70$ & Liquiflex $\mathrm{H}$ & incolor & flexível & transparente & não & não & $\operatorname{sim}$ \\
\hline $30 / 70$ & $30 / 70$ & R-45HTLO & incolor & flexível & transparente & não & não & $\operatorname{sim}$ \\
\hline $50 / 50$ & $0 / 100$ & - & incolor & $* *$ & transparente & não & não & $\operatorname{sim}$ \\
\hline $50 / 50$ & $30 / 70$ & Liquiflex H & incolor & flexível & transparente & não & não & $\operatorname{sim}$ \\
\hline $50 / 50$ & $30 / 70$ & R-45HTLO & incolor & flexível & transparente & não & não & $\operatorname{sim}$ \\
\hline \multicolumn{9}{|c|}{ Razão NCO/OH = 3} \\
\hline $30 / 70$ & $0 / 100$ & - & incolor* & flexível & transparente & não & não & $\operatorname{sim}$ \\
\hline $30 / 70$ & $30 / 70$ & Liquiflex H & incolor & flexível & transparente & não & não & $\operatorname{sim}$ \\
\hline $30 / 70$ & $30 / 70$ & R-45HTLO & incolor & flexível & transparente & não & não & $\operatorname{sim}$ \\
\hline $50 / 50$ & $0 / 100$ & - & incolor & flexível & transparente & não & não & $\operatorname{sim}$ \\
\hline $50 / 50$ & $30 / 70$ & Liquiflex $\mathrm{H}$ & incolor & flexível & transparente & não & não & $\operatorname{sim}$ \\
\hline $50 / 50$ & $30 / 70$ & R-45HTLO & incolor & flexível & transparente & não & não & $\operatorname{sim}$ \\
\hline \multicolumn{9}{|c|}{ Razão NCO/OH = 4} \\
\hline $30 / 70$ & $0 / 100$ & - & incolor* & rígida & transparente & não & não & não \\
\hline $30 / 70$ & $30 / 70$ & Liquiflex $\mathrm{H}$ & incolor & rígida & transparente & não & não & não \\
\hline $30 / 70$ & $30 / 70$ & R-45HTLO & incolor & rígida & transparente & não & não & não \\
\hline $50 / 50$ & $0 / 100$ & - & \multicolumn{6}{|c|}{ Dispersão instável com sedimentação } \\
\hline $50 / 50$ & $30 / 70$ & Liquiflex H & incolor* & flexível & transparente & não & não & $\operatorname{sim}$ \\
\hline $50 / 50$ & $30 / 70$ & R-45HTLO & incolor & flexível & transparente & não & não & $\operatorname{sim}$ \\
\hline
\end{tabular}

*levemente amarelado se comparado a um filme de polietileno; e ** muito flexível - alta pegajosidade (tack). 


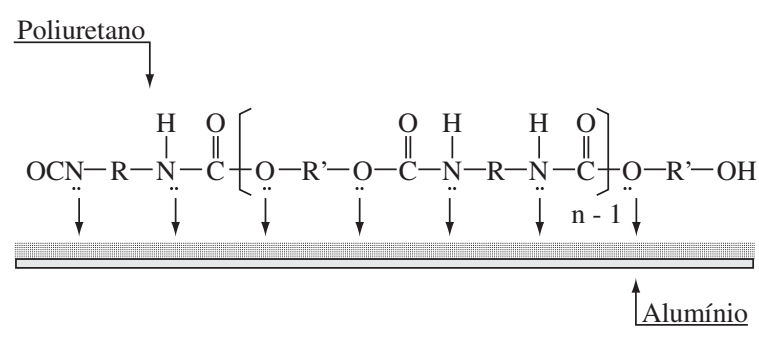

Figura 1. Representação esquemática da interação entre uma cadeia poliuretânica e a superfície de alumínio.

dos filmes que ficaram quebradiços, de razão $\mathrm{NCO} / \mathrm{OH}=4$ e relação DMPA/Poliol = 70/30, portador de maior quantidade de ligações uretânicas e ureicas. Os filmes apresentaram rigidez em função do elevado teor de segmentos rígidos, provenientes do diisocianato e do extensor de cadeia (hidrazi-

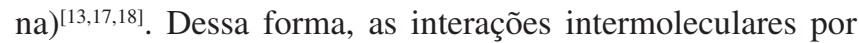
ligações de hidrogênio se tornam mais significativas entre as cadeias poliméricas, diminuindo a aderência ao substrato, já que os elétrons livres ficam menos disponíveis.

\section{Propriedades mecânicas dos filmes}

As Tabelas 9 e 10 apresentam os resultados dos ensaios mecânicos aos quais os filmes das Séries 1 e 2 foram submetidos. Os resultados de tensão de ruptura e alongamento dos filmes produzidos a partir das dispersões preparadas nas Séries 1 e 2 não apresentaram diferenças significativas, seguindo o comportamento observado também nos resultados obtidos por meio das outras técnicas de caracterização.

Pode-se verificar que, para os filmes vazados a partir das formulação obtidas sem o HTPB, o alongamento na ruptura foi ligeiramente maior do que o obtido para as formulações

Tabela 9. Valores de tensão na ruptura $(\sigma)$ e alongamento na ruptura $(\varepsilon)$ obtidos para os filmes da Série 1.

\begin{tabular}{|c|c|c|c|c|}
\hline \multirow{2}{*}{$\begin{array}{c}\text { Relação } \\
\text { DMPA/Poliol } \\
(\% / \%)\end{array}$} & \multirow{2}{*}{$\begin{array}{c}\text { Relação } \\
\text { HTPB/PPG } \\
(\% / \%)\end{array}$} & \multirow{2}{*}{$\begin{array}{l}\text { Tipo de } \\
\text { HTPB }\end{array}$} & \multicolumn{2}{|c|}{ Razão 2} \\
\hline & & & $\begin{array}{c}\sigma \\
(\mathbf{M P a})\end{array}$ & $\begin{array}{c}\varepsilon \\
(\%)\end{array}$ \\
\hline $70 / 30$ & $0 / 100$ & - & 18 & 742 \\
\hline $70 / 30$ & $10 / 90$ & Liquiflex $\mathrm{H}$ & 20 & 622 \\
\hline $70 / 30$ & $30 / 70$ & Liquiflex $\mathrm{H}$ & 19 & 662 \\
\hline $70 / 30$ & $10 / 90$ & Liquiflex $P$ & 18 & 730 \\
\hline $70 / 30$ & $30 / 70$ & Liquiflex P & 20 & 642 \\
\hline
\end{tabular}

em presença de HTPB. Pode-se observar que, com menor teor de DMPA, e conseqüente aumento no teor de polióis $($ DMPA/Polióis $=50 / 50)$, a tensão de ruptura sofreu uma diminuição significativa e o alongamento percentual foi recuperado nas formulações nas quais o HTPB foi adicionado.

Os resultados para as duas diferentes relações DMPA/Poliol das dispersões preparadas com razão $\mathrm{NCO} / \mathrm{OH}=2$, levam a concluir que o DMPA (neutralizado), além de promover a dispersão das partículas na fase aquosa, também é responsável por promover uma maior interação intermolecular no filme de poliuretano, melhorando a sua resistência à ruptura. $\mathrm{O}$ aumento nos valores de alongamento eram esperados para as dispersões preparadas com relação DMPA/Poliol $=50 / 50$, em conseqüência do maior teor de segmentos flexíveis ${ }^{[13,17,18]}$, provenientes do HTPB e do PPG adicionados nessas formulações.

Os resultados de propriedades mecânicas, dos filmes obtidos a partir das dispersões produzidas com razão $\mathrm{NCO} / \mathrm{OH}=3$, mostram que, para a relação DMPA/Poliol $=70 / 30$, a resistência mecânica foi ligeiramente afetada e o alongamento foi reduzido de forma acentuada. Esse comportamento é a consequiência da adição de maiores teores de diisocianato, responsável pelo aumento do teor de segmentos rígidos, que tornou os poliuretanos mais quebradiços. Para os filmes produzidos com relação DMPA/Polióis $=50 / 50$, foi possível observar a recuperação da resistência mecânica em conseqüência do maior teor de polióis, que associado ao alto teor de diisocianato $(\mathrm{NCO} / \mathrm{OH}=3)$, promoveu uma compensação. Isso permitiu que os valores obtidos com essa composição, se aproximassem dos valores observados para as formulações produzidas com razão $\mathrm{NCO} / \mathrm{OH}=2$ e relação DMPA/Polióis $=70 / 30$.

Os filmes obtidos a partir das dispersões preparadas com razão $\mathrm{NCO} / \mathrm{OH}=4$ não apresentaram resultados satisfatórios, dada a rigidez das cadeias. Os poliuretanos produzidos com razão DMPA/Poliol = 70/30 ficaram muito rígidos em virtude do alto teor de diisocianato usado. Os filmes após a secagem ficavam totalmente trincados, impossibilitando a realização de qualquer ensaio. Para as formulações obtidas em presença de HTPB, ocorreu um melhor equilíbrio entre as fases amorfa e cristalina, o que resultou na recuperação da resistência mecânica e também da capacidade elástica do material.

Tabela 10. Valores de tensão na ruptura $(\sigma)$ e alongamento na ruptura $(\varepsilon)$ obtidos para os filmes da Série 2.

\begin{tabular}{|c|c|c|c|c|c|c|c|c|}
\hline \multirow{2}{*}{$\begin{array}{c}\text { Relação } \\
\text { DMPA/Poliol } \\
(\% / \%)\end{array}$} & \multirow{2}{*}{$\begin{array}{c}\text { Relação } \\
\text { HTPB/PPG } \\
(\% / \%) \\
\end{array}$} & \multirow[t]{2}{*}{ Tipo de HTPB } & \multicolumn{2}{|c|}{ Razão 2} & \multicolumn{2}{|c|}{ Razão 3} & \multicolumn{2}{|c|}{ Razão 4} \\
\hline & & & $\begin{array}{c}\sigma \\
(\mathbf{M P a}) \\
\end{array}$ & $\begin{array}{c}\varepsilon \\
(\%)\end{array}$ & $\begin{array}{c}\sigma \\
(\mathbf{M P a})\end{array}$ & $\begin{array}{c}\varepsilon \\
(\%)\end{array}$ & $\begin{array}{c}\sigma \\
(\mathbf{M P a})\end{array}$ & $\begin{array}{c}\varepsilon \\
(\%)\end{array}$ \\
\hline $30 / 70$ & $0 / 100$ & - & 18 & 742 & 11 & 240 & quebrou & quebrou \\
\hline $30 / 70$ & $30 / 70$ & Liquiflex H & 19 & 662 & 12 & 65 & quebrou & quebrou \\
\hline $30 / 70$ & $30 / 70$ & R-45HTLO & 16 & 544 & 16 & 31 & quebrou & quebrou \\
\hline $50 / 50$ & $0 / 100$ & - & tack & tack & 14 & 858 & ND & ND \\
\hline $50 / 50$ & $30 / 70$ & Liquiflex $\mathrm{H}$ & 11 & 931 & 19 & 602 & 16 & 292 \\
\hline $50 / 50$ & $30 / 70$ & R-45HTLO & 12 & 938 & 17 & 545 & 13 & 231 \\
\hline
\end{tabular}

ND- Não determinado. Sedimentação 24 h após a síntese. 


\section{Conclusão}

As condições de síntese descritas neste trabalho permitiram a obtenção de dispersões aquosas à base de poliuretanos que apresentaram estabilidade frente à sedimentação, e variações mínimas nos valores de sólidos totais, viscosidade aparente e tamanho médio de partícula, independente da formulação. Foi possível produzir formulações não-poluentes com viscosidade adequada para aplicação como revestimentos, em ausência de qualquer solvente orgânico e catalisador e com propriedades mecânicas satisfatórias.

\section{Agradecimentos}

Ao CNPq, FAPERJ, CAPES pelo apoio financeiro, ao IAE/CTA pela doação do diisocianato e à Petroflex Indústria e Comércio pela doação dos polibutadienos hidroxilados e por permitir o desenvolvimento deste estudo em seus laboratórios.

\section{Referências Bibliográficas}

1. Leonardão, E. J.; Freitag, R. A.; Dabdoub, M. J.; Batista, A. C. F. \& Silveira, C. C. - Química. Nova, 26, p.123 (2003).

2. Sanderson, R. D.; Cloete, V.; Seboa, S. \& Karmis, A. - "Green technology, preparation of moisture proof polymer coatings that leave paper recyclable", in: 5th Annual UNESCO School and IUPAC Conference on Macromolecules \& Materials Science, p.23, Stellenbosch, South Africa. Mar (2002).

3. Coutinho, F. M. B.; Delpech, M. C. \& Garcia, M. E. F. - Polímeros: Ciência e Tecnologia, 4, p.230 (2004).

4. Coutinho, F. M. B.; Delpech, M. C.; Alves, T. L. \& Ferreira, A. A. - Polymer Degradation and Stability, 81, 1, p.19-27, 2003.

5. Coutinho, F. M. B., Delpech, M. C. \& Alves, T. L. - Polímeros: Ciência e Tecnologia, 12, p.248 (2002).

6. Coutinho, F. M. B.; Delpech, M. C. \& Garcia, M. E. F. - Polymer Testing, 21, p.719 (2002).

7. Coutinho, F. M. B.; Delpech, M. C.; Alves, L. S. - Journal of Applied Polymer Science, 80, p.566 (2001).

8. Coutinho, F. M. B. \& Delpech, M. C. - Polymer Degradation and Stability, 70, p.49 (2000).

9. Coutinho, F. M. B.; Delpech, M. C. \& Alves, L. S - Polímeros: Ciência e Tecnologia, 10, p. 49 (2000).
10. Delpech, M. C. \& Coutinho, F. M. B. - Polymer Testing, 19, p.939 (2000).

11. Delpech, M. C. \& Coutinho, F. M. B. - Polímeros: Ciência e Tecnologia, 9, p. 41 (1999).

12. Coutinho, F. M. B.; Alves, L. S. \& Delpech, M. C. Anais da Associação Brasileira de Química, 47, p.255 (1998).

13. Coutinho, F. M. B. \& Delpech, M. C. - Polymer Testing, 15, p.103 (1996).

14. Delpech, M. C.; Coutinho, F. M. B.; Moura, P. M. Q. \& Mello, S. D. S. - Polímeros: Ciência e Tecnologia, 3, p.30 (1996).

15. Jang, J. Y.; Jhon, Y. K.; Cheong, I. W. \& Kim, J. H. Physicochemical and Engineering Aspects, 196, p.135 (2002).

16. Pavlova, M.; Draganova, M. \& Novakov, P. - Polymer, 26, p.1901 (1985).

17. Hepburn, C. - "Polyurethane elastomers", second edition, Elsevier Science Publishers Ltd., London (1991).

18. Bamford, C. H. \& Eastmond, G. C. - "Polyuretnane Block Copolymers", in: Encyclopedia of Polymer Science and Engineering. Mark, H. F.; Bikales, N. M.; Overberger, C. G. \& Mengens, G. (ed), Jonh Wiley \& Sons, Ney York (1985).

19. Dow Chemical Company, Products information, Dow Plastic, Polyether Polyol Voranol 2120, Disponível em: $<$ http://www.dow.com>. Acesso em 01/03/2006.

20. Petroflex Indústria e Comercio Ltda., Família de produtos, Liquiflex, Disponível em: http://www.flexglobal. com.br/web/pt/produtos/liquiflex.htm. Acesso em: 01/04/2006.

21. Sartomer Company Inc., Technical Data Sheet: Poly BD $^{\circledR}$ R-45HTLO, Disponível em: http://www.sartomer.com/wpapers/2250.pdf. Acesso em: 01/04/2006.

22. -"Book of American Society for Testing and Materials Standards - ASTM", D 412-68, Easton, 1979.

23. Kim, C. K. \& Kim, B. K. - J. Appl. Polym. Sci., 43, p.2295 (1991).

24. Kim, T. K. \& Kim, B. K. - Colloid Polym. Sci., 269, p.889 (1991).

Enviado: 01/01/07

Reenviado: $10 / 04 / 07$

Aceito: $20 / 04 / 07$ 